\title{
Opportunities for developing therapies for rare genetic diseases: focus on gain-of- function and allostery
}

\author{
Binbin Chen ${ }^{1}$ and Russ B. Altman ${ }^{1,2^{*}}$
}

\begin{abstract}
Background: Advances in next generation sequencing technologies have revolutionized our ability to discover the causes of rare genetic diseases. However, developing treatments for these diseases remains challenging. In fact, when we systematically analyze the US FDA orphan drug list, we find that only $8 \%$ of rare diseases have an FDA-designated drug. Our approach leverages three primary insights: first, diseases with gain-of-function mutations and late onset are more likely to have drug options; second, drugs are more often inhibitors than activators; and third, some disease-causing proteins can be rescued by allosteric activators in diseases due to loss-of-function mutations.
\end{abstract}

Results: We have developed a pipeline that combines natural language processing and human curation to mine promising targets for drug development from the Online Mendelian Inheritance in Man (OMIM) database. This pipeline targets diseases caused by well-characterized gain-of-function mutations or loss-of-function proteins with known allosteric activators. Applying this pipeline across thousands of rare genetic diseases, we discover 34 rare genetic diseases that are promising candidates for drug development.

Conclusion: Our analysis has revealed uneven coverage of rare diseases in the current US FDA orphan drug space. Diseases with gain-of-function mutations or loss-of-function mutations and known allosteric activators should be prioritized for drug treatments.

Keywords: Genetic diseases, Rare diseases, Orphan drugs, Drug targets, Gain-of-function, Allosteric, Drug discovery

\section{Background}

Rare diseases are defined as diseases affecting fewer than 200,000 patients in the US or fewer than 1 in 2000 people in the EU; over 25 million Americans suffer from at least one of 7000 rare diseases [1]. Due to the limited market size and cost of drug development, the development of rare disease treatments continues to be challenging for pharmaceutical companies, despite incentives created in the 1983 US Orphan Drug Act [2]. By obtaining orphan drug status, a pharmaceutical company can gain regulatory benefits including application fee waivers and extended time for market protection. Thus the US FDA orphan drug list is a barometer for the current treatment development for rare diseases. In this study,

\footnotetext{
* Correspondence: rbaltman@stanford.edu
${ }^{1}$ Department of Genetics, Stanford University School of Medicine, Stanford,

'Department of Genetics, Stanford University School of Medicine, Stanford, CA, USA

2Department of Bioengineering, Stanford University School of Medicine, Stanford, CA, USA
}

we examine the list of current and anticipated orphan drugs, and extract trends that may suggest other opporunities for development of useful therapies.

Many rare diseases have been linked to genetic abnoralities, and next-generation sequencing has accelerated ability to make such links [3]. It took more than years to identify the cystic fibrosis (MIM: 219700) ing gene CFTR by chromosomal walking in 1980 In contrast, with next-generation sequencing and few years [5]. The Online Mendelian Inheritance in Man (OMIM) curates both genetic and clinical information about rare diseases caused by single mutations [6]. Single mutation diseases have better understood pathological mechanisms, which is critical for drug development [7]. We therefore use the OMIM as the basis of our search for rare disease targets. 
Most small molecule drugs inhibit their targets [8]. When a protein structure is altered, gain-of-function changes are more easily modulated by small molecules than loss-of-function changes; it is more difficult to rescue function. Not surprisingly, the field has had more success developing antagonists than agonists. For example, Drugbank, one of the most commonly used drug databases, includes more than 1700 small molecule inhibitors or antagonists, but only 423 small molecule activators or agonists [9].

Solved protein three-dimension (3D) structures provide a molecular basis for understanding the implications of coding variations on protein conformation, and enable rational drug design [10-12]. Thus, our study focuses on the subset of potential drug targets with both gain-offunction mutations and available 3D protein structures.

In brief, we have built a pipeline (Fig. 1) to search for small molecule drug development opportunities among rare genetic diseases based on the following three assumptions. First, the disease target should be caused by a single gain-of-function mutation, so we can focus on inhibiting a single disease-driver protein rather than multiple pathways. Second, the disease should have late or adult onset, which provides a large time window to introduce therapies. Finally, the primary disease gene product should have a solved crystal structure, which is desirable for rational-based inhibitor design. Although these limit the scope of our analysis, they provide a clear rational for moving forward when the criteria are met.

Our pipeline also supports targeting diseases due to loss-of-function mutations with a known allosteric activator (Fig. 1). Allosteric regulation is a common feature in enzymatic activity. In some cases, an allosteric activator can increase the activity of a mutated enzyme, moving it towards a more physiologically normal range [13]. For example, N-carbamylglutamate (carglumic acid) can treat carbamyl phosphate synthetase I (CPSI) deficiency (MIM:237300) due to its ability to activate CPSI via an allosteric site [14] N-carbamylglutamate was approved by the FDA in 2010 [15]. The Allosteric Database (ASD) provides protein and allosteric modulator pairs that may be useful in diseases due to loss-offunction mutations [16].

\section{Methods}

\section{Overview of pipeline to search for drug targets}

We demonstrate the overall pipeline in the Fig. 1. We downloaded the complete OMIM database including mutated genes and disease descriptions in June 2015 [6]. Only diseases with known mutations are considered in our analysis.

First, we obtained a list of the potential diseases due to gain-of-function mutations by filtering for any OMIM disease entries mentioning gain-of-function related terms (Additional file 1: Table S1). We assumed the rest of diseases are diseases due to loss-of-function mutations. For each subset of diseases, we filtered them with our selection criteria discussed below to generate the final candidate list.

\section{Searching for gain-of-function rare genetic diseases}

To become candidates, all diseases due to gain-offunction mutations (Additional file 1: Table S7) must

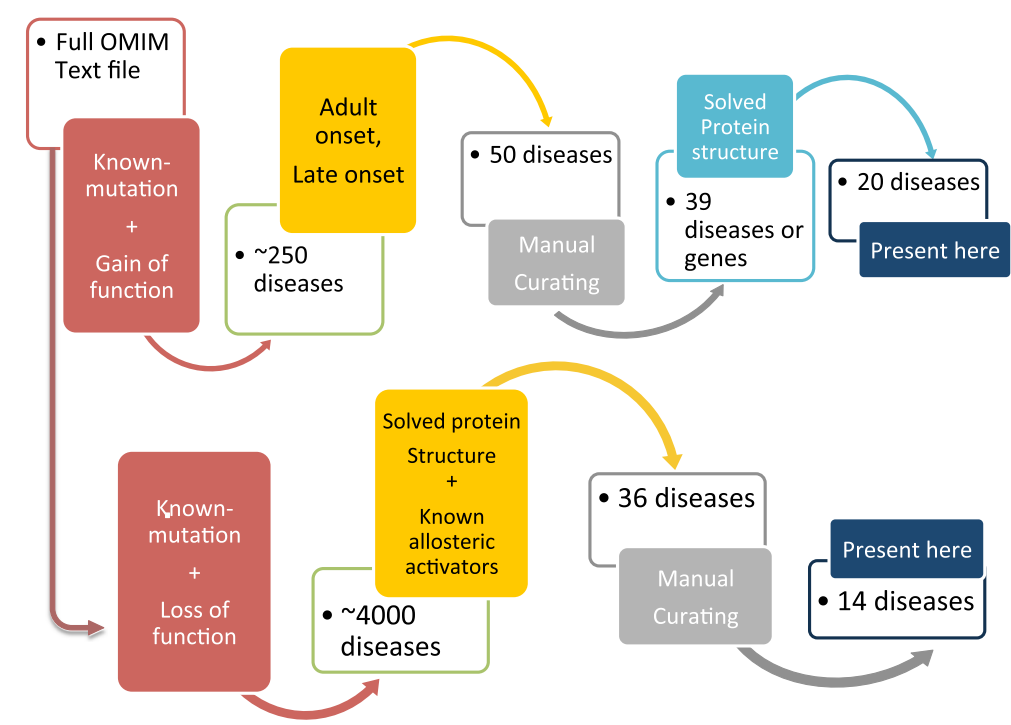

Fig. 1 Texting mining algorithm to search for targetable rare diseases. We filtered all Mendelian diseases with known mutated genes in the OMIM for gain-of-function and late clinical onset related terms for the fist step of filtering to determine our disease targets. In a parallel branch of the pipeline, we filtered for diseases due to loss-of-function mutations with known allosteric activators. All candidates must have a solved protein structure. We manually verified the final disease list to ensure each disease mechanism and onset match our computationally generated label 
have late clinical onset and their mutated proteins need to have solved crystal structures. To determine whether a disease has late onset, we required at least one related term (Additional file 1: Table S9) to appear in the OMIM disease description section. We determined crystal structure availability with the Gene ID provided by the OMIM and the UniProt mapping tool [17]. To reduce false positives, we manually verified all potential late onset diseases due to gain-of-function mutations. Structure proteins are unlikely to be inhibited by small molecule drugs given their abundance. We manually excluded diseases caused by structure proteins from the filtered disease list using the list in Additional file 1: Table S10.

\section{Searching for treatable rare genetic diseases due to loss-of-function mutations}

For each disease due to loss-of-function mutations from the previous step (Fig. 1), we obtain its gene ID from the OMIM. We queried the Allosteric Database 2.0 (ASD) [16] for these gene IDs to check whether a small molecule can activate each disease's mutated proteins. Using the Uniprot mapping tool, we then checked whether these gene IDs have a solved crystal structure for their gene product. In some cases, the OMIM includes weak links between mutated genes and diseases. Thus, for the final set of candidates, we manually verified strong evidence supporting the mechanism of each disease.

\section{Mapping the FDA orphan drug list to targeted diseases and disease categories}

We obtained the list of FDA approved and designated orphan drugs from the FDA website in July 2015 [18]. The FDA provides a treatment description for each orphan drug. For example, for alglucerase, the treatment description is: "for replacement therapy in patients with Gaucher's disease type I." From each of these treatment descriptions, we extracted all disease names that appear in the Comparative Toxicogenomics Database (CTD), e.g., "Gaucher's disease" (MIM: 230800). The CTD groups diseases into categories that capture high-level characteristics such as mechanism or organ system (e.g., Genetic, Musculoskeletal). We connected each orphan drug with these CTD categories through the diseases mentioned in its treatment description, and counted which categories are most often targeted. The CTD also links genetic diseases with their OMIM identifiers. We used these identifiers to connect each orphan drug with the diseases due to gainof-function mutations discovered in the previous step.

\section{Results}

Orphan drugs cover a small portion of rare diseases

The current FDA approved and designated orphan drugs target 243 and 597 rare disease related conditions, respectively (Fig. 2, Additional file 1: Tables S1, S2). This is still a very small portion of 7000 rare diseases [19] since only $8 \%$ of rare diseases have any designated orphan drugs.

We observe uneven coverage; cancer, neurological disorders, and genetic diseases are the most common disease categories (Fig. 3). 16-18\% of the current targeted diseases are genetic diseases (Additional file 1: Tables S3, S5). Genetic diseases are underrepresented since over $60 \%$ of the known rare diseases are Mendelian diseases [6]. In comparison, 30-38\% of orphan drugs target rare cancers, defined as affecting fewer than 200,000 patients (Fig. 3, Additional file 1: Tables S3, S5).

\section{Gain-of-functions and targetability of the disease}

$6 \%$ of all Mendelian disorders have a designated orphan drug. In comparison, $12 \%$ of gain-of-function of Mendelian subsets have a designated orphan drug (Fig. 4). This number goes up to $16 \%$ when we further filter for adult onset diseases (Fig. 4), which supports our assumptions that diseases due to gain-of-function mutations are more readily treated with small molecules drugs.

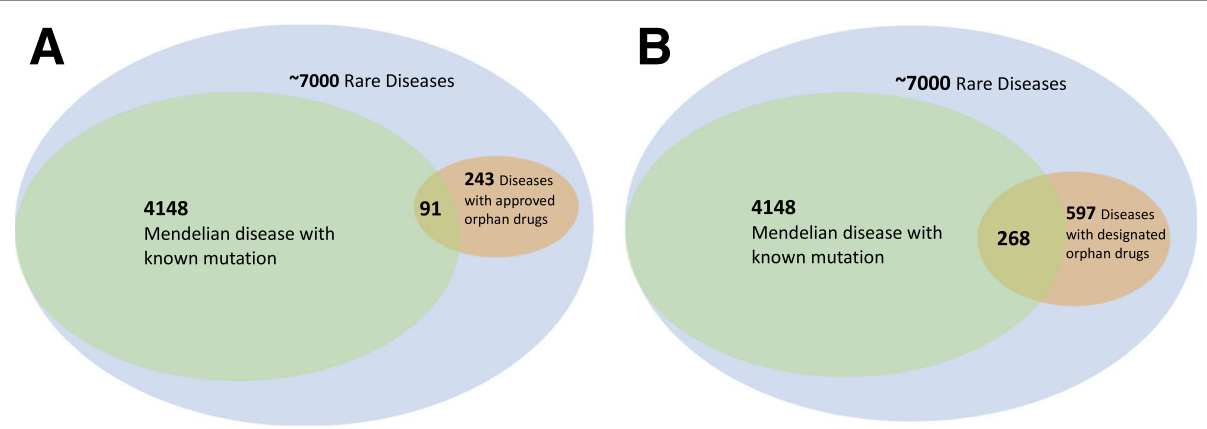

Fig. 2 Overlap diagrams for the current approved or designated orphan drug space with the rare disease space. Each FDA approved and designated orphan drug was linked to a disease and potential OMIM ID based on the CTD table. 243 and 597 rare diseases are covered by the approved (a) and designed (b) orphan drugs. The current treatment space covers a small fraction of the rare disease space 


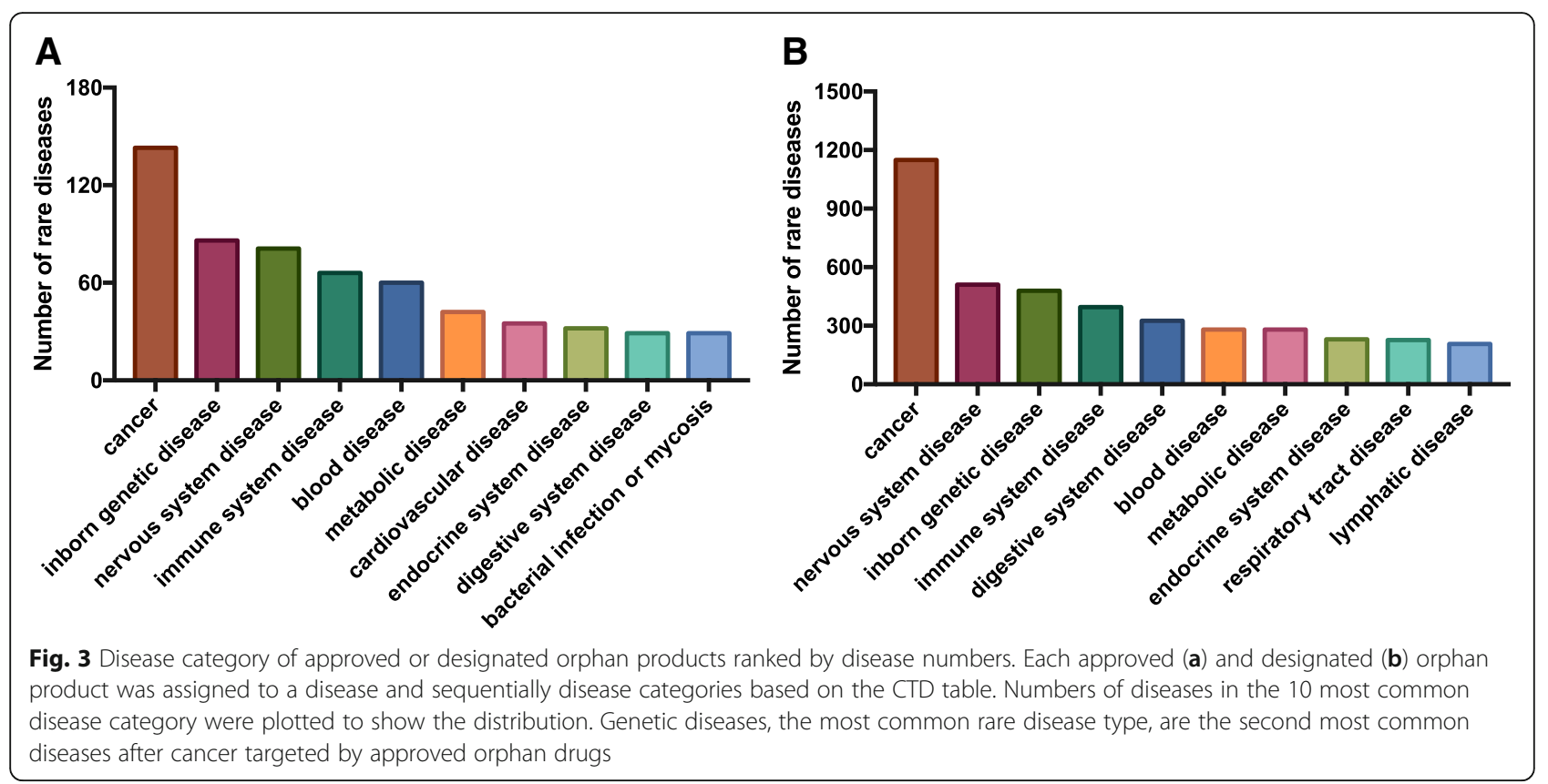

\section{Promising targets}

Tables 1 and 2 show the top disease candidates due to gain-of-function and loss-of-function mutations and their associated OMIM IDs and mutated genes. We group gain-of-function mutation diseases based on their molecular mechanism. No obvious pathophysiology groups are

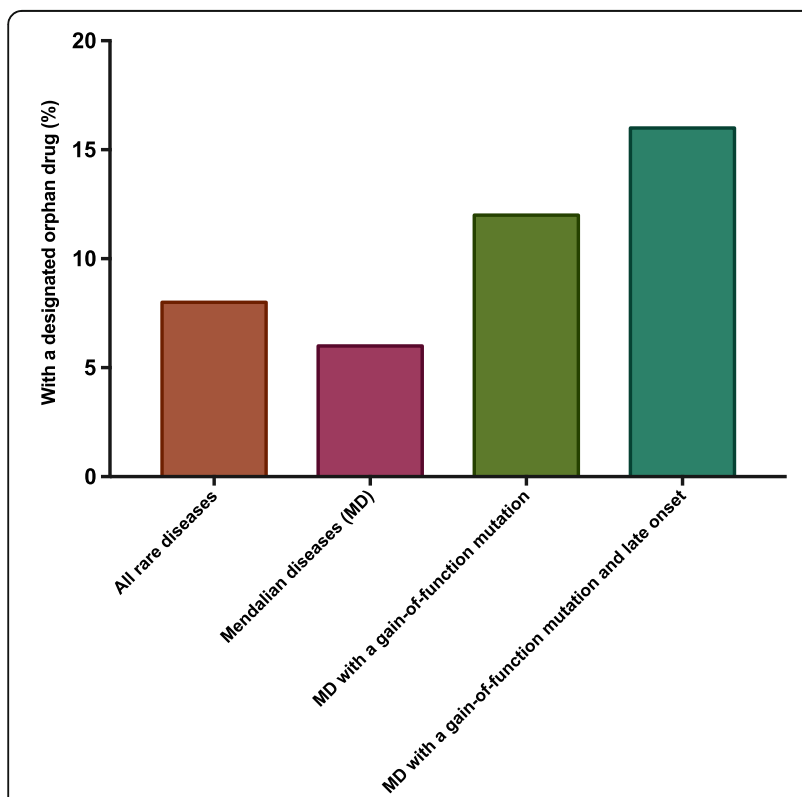

Fig. 4 Diseases with gain-of-function mutations have higher chance to have treatment under development. Percentages of rare diseases covered by at least one FDA-designated orphan drug were plotted across categories. Mendelian diseases with a gain-of-function mutation and late clinical onset has the highest chance to be readily covered by a orphan drug (16 vs. $8 \%$ for all rare diseases) present in these diseases caused by loss-of-function mutations. Additional file 1: Tables S7 and S8 list all gain-of-function and loss-of-function mutation candidates passed our computational filter.

\section{Discussion}

Substantial opportunities and challenges remain in the current treatment development for rare genetic diseases, since $92 \%$ of rare diseases lack FDA-designated products. In 2015, 243 diseases have at least one approved orphan drug, a small increase compared to the 200 diseases reported in 2010 [2]. Our analysis shows that diseases due to gain-of-function mutations have more orphan drug designations, and thus may be good targets for drug discovery programs.

Our disease candidates for gain-of-function mutations in Table 1 include known over-activation diseases with inhibitor drug developments on trial, like PRPS1 superactivity (MIM: 300661) and achondroplasia (MIM: 100800) [20, 21]. The majority of gain-of-function disease candidates in Table 1 are neuromuscular diseases, such as spinocerebellar ataxia (MIM: 183086, $183090,109150,183086,164500,607136)$ or the overactivation of neuron ion channels. This is consistent with the fact that neurological disorders are one of the most common disease categories in the current FDA orphan drug list.

It is striking that over half of the diseases in Table 1 are caused by long trinucleotide repeats including both CAG and non-CAG repeats. In fact, this list contains seven out of nine known CAG repeat disorders [22]. This is because long trinucleotide repeats cause gain- 
Table 1 Top targetable diseases due to gain-of-function mutations

\begin{tabular}{|c|c|c|c|}
\hline Type & Diseases & OMIM & Gene/Protein \\
\hline \multirow[t]{6}{*}{ Over-activation } & $\begin{array}{l}\text { Hereditary motor and sensory neuropathy } \\
\text { type IIC }\end{array}$ & 606071 & TRPV4 \\
\hline & $\begin{array}{l}\text { Postsynaptic slow-channel congenital } \\
\text { myasthenic syndrome }\end{array}$ & 601462 & CHRNA1, CHRND, CHRNE, CHRNB1 \\
\hline & PRPS1 superactivity & 300661 & PRPS1 \\
\hline & Parkinson disease-8 & 607060 & LRRK2 \\
\hline & Tubular aggregate myopathy & 160565 & STIM1 \\
\hline & Achondroplasia & 100800 & FGFR3 \\
\hline \multirow[t]{2}{*}{ Gene duplication } & Lubs X-linked mental retardation syndrome & 300260 & MECP2 \\
\hline & Parkinson disease- 4 & 605543 & SNCA \\
\hline \multirow[t]{3}{*}{ CAG repeat } & Spinocerebellar ataxia 1,2,3,6,7,17 & $\begin{array}{l}\text { 183086, 183090, 109150, } \\
183086,164500,607136\end{array}$ & ATXN1,2,3, CACNA1A,ATXN7, TBP \\
\hline & Huntington & 6066438 & HTT \\
\hline & Spinal and bulbar muscular atrophy $X$-linked & 313700 & $A R$ \\
\hline \multirow[t]{4}{*}{ Non-CAG trinucletide repeat } & Friederich Ataxia 1 & 229300 & FXN \\
\hline & Myotonic Dystrophy 1 & 160900 & DMPK \\
\hline & Oculopharyngeal muscular dystrophy & 164300 & PABPN1 \\
\hline & Spinocerebellar ataxia 8 & 608768 & ATXN8 \\
\hline
\end{tabular}

Corresponding disease names, OMIM identifiers, and mutated genes are listed. Diseases are grouped by the molecular mechanism as indicated in the type column. The rest of potential candidates can be found in Additional file 1: Table S7

of-function toxicity with late onset. Recently, drugs inhibiting the pathway involved in expressing trinucleotide repeat regions have drawn attention from the field. For example, DRB Sensitivity Inducing Factor (DSIF), which is comprised of SUPT4H1 and SUPT5H, is essential to expressing long CAG repeats in vitro and in vivo $[23,24]$. A small molecule that selectively inhibits DSIF would be a potential treatment for trinucleotide repeat disorders.

A small number of Mendelian diseases with a loss-offunction mutation have intrinsic allosteric activators. Only 36 diseases passed stringent our filter, a small fraction of the 571 total diseases curated in the ASD (Fig. 1, Additional file 1: Table S8) [16]. This is because

Table 2 Top targetable genetic diseases due to loss-of-function mutations

\begin{tabular}{llll}
\hline Disease name & OMIM & Gene/Protein & Allosteric activators \\
\hline Brugada syndrome 3 & 611875 & CACANA1C & BayK 8644, FPL64176 \\
Congenital amegakaryocytic thrombocytopenia & 604498 & MPL & PF \\
Frontal lobe nocturnal epilepsy 3 & 605375 & CHRNB2 & Desformylflustrabromine and others \\
Glycogen storage disease V & 232600 & PYGM & AMP and IMP \\
Glycogen storage disease VI & 232700 & PYGL & AMP and IMP \\
Hereditary pancreatitis & 167800 & CTRC & BisQ, Bis Q Benzyl \\
Homocystinuria due to cystathionine beta-sythease deficiency & 236200 & CBS & SAM \\
Hyperekplexia hereditary 1 & 149400 & GLRA1 & Ajulemic acid, Trifluoroacetate \\
Isolated growth hormone deficiency type III & 307200 & BTK & PIP3 \\
Lung cancer susceptibility & 612052 & CHRNA3 & rac-12 k, rac-14e \\
Muscle glycogen storage disease 0 & 611556 & GYS1 & Glc6P \\
Ovarian dysgenesis 1 & 233300 & FSHR & Ajulemic acid, Trifluoroacetate \\
Pigmented nodular adrenocortical disease & 610475 & PDE11A & Estradiol \\
Thrombocysthemia 2 & 601977 & MPL & PF
\end{tabular}

Corresponding disease names, OMIM identifiers, mutated genes and known allosteric activators are listed. Allosteric activators are queried from the Allosteric Database. The rest of potential candidates can be found in Additional file 1: Table S8 
we require both an allosteric activator and a solved human protein crystal structure for the protein.

Based on FDA Rare Disease Repurposing Database [18], about 525 out of 2300 orphan drug designations in 2010 were based on drug-repurposing. Drug repurposing will continue to be an important route for orphan drug development given the substantial reduction in cost and time. Disease genes/proteins listed in the Table 1 and 2 can be evaluated against the current FDA-approved drug list to determine if any available drugs can inhibit the gain-of-function proteins or activate the loss-of-function proteins $[9,25,26]$.

In this analysis we are targeting the driver mutations of rare diseases; we have not expanded our search algorithm to their upstream regulator and downstream effectors, although these are certainly worth examining [27]. The results from this study can be integrated with other rare disease research platforms like RD-Connect [28] and Orphanet [29-31] to further collaborations across disciplines and institutes.

\section{Conclusion}

Here we present an evaluation of the current FDA orphan drug space and opportunities for treatment developments in the rare disease domain. Our pipeline provides a list of potential low-hanging fruit for orphan drug research. Drug development programs that are effective at finding inhibitors could focus on the gain-offunction candidates. Known allosteric modulators could be screened against the loss-of-function candidates to quickly evaluate the opportunities for moving forward.

\section{Additional file}

Additional file 1: Table S1. The disease mapping for the current FDA-approved orphan drugs. Table S2. The disease mapping for the current FDA-designated orphan drugs. Table S3. The disease category mapped for the current FDA-approved orphan drugs ranked by drug number. Table S4. The individual diseases mapped for the current FDA-approved orphan drugs ranked by drug number. Table S5. The disease category mapped for the current FDA-designated orphan drugs ranked by drug number. Table S6. The individual diseases mapped for the current FDA-designated orphan drugs ranked by drug number. Table S7. The complete text-mining results of the OMIM for diseases caused by a single gain-of-function mutation. Table $\mathbf{S 8}$. The complete text-mining results of the OMIM for diseases caused by a single loss-of-function mutation and associated with a potential allosteric activator. Table S9. Terms used to determine gain-of-function and late disease onset in the OMIM disease description. Table S10. Terms used to manually determine if a gain-of-function disease is caused by a mutated structure protein. (ZIP $357 \mathrm{~kb})$

\section{Abbreviations}

ASD: The Allosteric Database; CPSI: Carbamyl phosphate synthetase I; CTD: Comparative Toxicogenomics Database; DSIF: DRB Sensitivity Inducing Factor; MIM: OMIM ID; OMIM: Online Mendelian Inheritance in Man Database

\section{Acknowledgements}

We thank Emily Mallory and Dr. Grace Tang for discussing ideas and assistance in text mining strategies. We thank Ethan Fast for giving feedbacks on the manuscript. This work is supported by the NIH/Stanford Medical Scientist Training Program grant and the PD Soros Fellowship.

\section{Funding}

BC is supported by the Stanford/NIH MSTP grant and PD Soros Fellowship. No funding body was directly involved in design, analysis, and publication of this study.

\section{Availability of data and materials}

Full analysis of the current FDA orphan drug list and the OMIM rare diseases can be found in the Additional file 1: Table S1-10. Further information can be provided upon request.

\section{Authors' contributions}

Both authors made substantial contributions to conception of the article, participated in drafting the main text and reviewed the final version. $\mathrm{BC}$ conducted most of the computational analysis. Both authors read and approved the final manuscript.

\section{Competing interest}

RBA is a scientific consultant to Personalis, Pfizer and Karius. BC declares no conflict of interest.

\section{Consent for publication}

Not applicable.

Ethics approval and consent to participate

Not applicable.

\section{Publisher's Note}

Springer Nature remains neutral with regard to jurisdictional claims in published maps and institutional affiliations.

Received: 10 December 2016 Accepted: 19 March 2017 Published online: 17 April 2017

\section{References}

1. Tambuyzer E. Rare diseases, orphan drugs and their regulation: questions and misconceptions. Nat Rev Drug Discov. 2010;9(12):921-9.

2. Braun MM, Farag-El-Massah S, Xu K, Coté TR. Emergence of orphan drugs in the United States: a quantitative assessment of the first 25 years. Nat Rev Drug Discov. 2010;9(7):519-22.

3. Wastfelt M, Fadeel B, Henter J. A journey of hope: lessons learned from studies on rare diseases and orphan drugs. J Intern Med. 2006;260(1):1-10.

4. Riordan JR, Rommens JM, Kerem B, Alon N, Rozmahel R, Grzelczak Z, Zielenski J, Lok S, Plavsic N, Chou JL, et al. Identification of the cystic fibrosis gene: cloning and characterization of complementary DNA. Science. 1989;245(4922):1066-73.

5. Cirulli ET, Goldstein DB. Uncovering the roles of rare variants in common disease through whole-genome sequencing. Nat Rev Genet. 2010;11(6):415-25.

6. Amberger J, Bocchini C, Hamosh A. A new face and new challenges for online mendelian inheritance in Man (OMIM(R)). Hum Mutat. 2011;32(5):564-7.

7. Thomas PD, Kejariwal A. Coding single-nucleotide polymorphisms associated with complex vs. Mendelian disease: evolutionary evidence for differences in molecular effects. Proc Natl Acad Sci U S A. 2004;101(43):15398-403.

8. Arkin MR, Wells JA. Small-molecule inhibitors of protein-protein interactions: progressing towards the dream. Nat Rev Drug Discov. 2004;3(4):301-17.

9. Law $V$, Knox C, Djoumbou Y, Jewison T, Guo AC, Liu Y, Maciejewski A, Arndt D, Wilson M, Neveu V, et al. DrugBank 4.0: shedding new light on drug metabolism. Nucleic Acids Res. 2014;42(Database issue):D1091-1097.

10. Truhlar DG, Howe WJ, Hopfinger AJ, Blaney J, Dammkoehler RE. Rational Drug Design, vol. 108. New York: Springer Science \& Business Media; 2012

11. Price MLP, Jorgensen WL. Rationale for the observed COX-2/COX-1 selectivity of celecoxib from Monte Carlo simulations. Bioorg Med Chem Lett 2001;11(12):1541-4. 
12. Jorgensen WL, Duffy EM. Prediction of drug solubility from structure. Adv Drug Deliv Rev. 2002;54(3):355-66.

13. Goodey NM, Benkovic SJ. Allosteric regulation and catalysis emerge via a common route. Nat Chem Biol. 2008:4(8):474-82.

14. Tuchman M, Caldovic L, Daikhin Y, Horyn O, Nissim I, Nissim I, Korson M, Burton B, Yudkoff M. N-carbamylglutamate markedly enhances ureagenesis in $\mathrm{N}$-acetylglutamate deficiency and propionic acidemia as measured by isotopic incorporation and blood biomarkers. Pediatr Res. 2008;64(2):213-7.

15. Haberle J. Role of carglumic acid in the treatment of acute hyperammonemia due to $\mathrm{N}$-acetylglutamate synthase deficiency. Ther Clin Risk Manag. 2011;7:327-32.

16. Huang Z, Mou L, Shen Q, Lu S, Li C, Liu X, Wang G, Li S, Geng L, Liu Y, et al. ASD v2.0: updated content and novel features focusing on allosteric regulation. Nucleic Acids Res. 2014;42(Database issue):D510-516.

17. UniProt C. UniProt: a hub for protein information. Nucleic Acids Res. 2015;43(Database issue):D204-212.

18. Xu K, Cote TR. Database identifies FDA-approved drugs with potential to be repurposed for treatment of orphan diseases. Brief Bioinform. 2011;12(4):341-5.

19. Schieppati A, Henter Jl, Daina E, Aperia A. Why rare diseases are an important medical and social issue. Lancet. 2008;371(9629):2039-41.

20. Laederich MB, Horton WA. FGFR3 targeting strategies for achondroplasia. Expert Rev Mol Med. 2012;14:e11.

21. de Brouwer AP, van Bokhoven H, Nabuurs SB, Arts WF, Christodoulou J, Duley J. PRPS1 mutations: four distinct syndromes and potential treatment. Am J Hum Genet. 2010;86(4):506-18.

22. Cummings $\mathrm{CJ}$, Zoghbi HY. Fourteen and counting: unraveling trinucleotide repeat diseases. Hum Mol Genet. 2000;9(6):909-16.

23. Liu CR, Chang CR, Chern Y, Wang TH, Hsieh WC, Shen WC, Chang CY, Chu IC, Deng N, Cohen SN, et al. Spt4 is selectively required for transcription of extended trinucleotide repeats. Cell. 2012;148(4):690-701.

24. Cheng HM, Chern Y, Chen IH, Liu CR, Li SH, Chun SJ, Rigo F, Bennett CF, Deng $N$, Feng $Y$, et al. Effects on murine behavior and lifespan of selectively decreasing expression of mutant huntingtin allele by supt $4 \mathrm{~h}$ knockdown. PLoS Genet. 2015;11(3):e1005043.

25. Lounkine E, Keiser MJ, Whitebread S, Mikhailov D, Hamon J, Jenkins JL, Lavan P, Weber E, Doak AK, Cote S, et al. Large-scale prediction and testing of drug activity on side-effect targets. Nature. 2012;486(7403):361-7.

26. Keiser MJ, Roth BL, Armbruster BN, Ernsberger P, Irwin JJ, Shoichet BK. Relating protein pharmacology by ligand chemistry. Nat Biotechnol. 2007;25(2):197-206.

27. Tsiliki G, Kossida S. Fusion methodologies for biomedical data. J Proteomics. 2011;74(12):2774-85.

28. Thompson R, Johnston L, Taruscio D, Monaco L, Beroud C, Gut IG, Hansson MG, 't Hoen PBA, Patrinos GP, Dawkins H, et al. RD-connect: an integrated platform connecting databases, registries, biobanks and clinical bioinformatics for rare disease research. J Gen Intern Med. 2014;29:5780-7.

29. Okumura T, Menez DA, Abayawickrama T. Refining disease databases for clinical decision support systems. Vol no. 00, p. 938-939. doi:10.1109/BIBM. 2015.7359809.

30. Ito M, Nakagawa S, Mizuguchi K, Okumura T. Integration of disease entries across OMIM, orphanet, and a proprietary knowledge base. Lect Notes Artif Intell. 2015;9101:120-30

31. Maiella S, Rath A, Angin C, Mousson F, Kremp O. Orphanet and its consortium: where to find expert-validated information on rare diseases. Rev Neurol France. 2013;169:S3-8.

\section{Submit your next manuscript to BioMed Central and we will help you at every step:}

- We accept pre-submission inquiries

- Our selector tool helps you to find the most relevant journal

- We provide round the clock customer support

- Convenient online submission

- Thorough peer review

- Inclusion in PubMed and all major indexing services

- Maximum visibility for your research

Submit your manuscript at www.biomedcentral.com/submit
Biomed Central 